\title{
CAN AN INEXPENSIVE PHONE APP COMPARE TO OTHER METHODS WHEN IT COMES TO 3D DIGITIZATION OF SHIP MODELS
}

\author{
M. Lewis and C. Oswald \\ Ship Reconstruction Laboratory, Nautical Archaeology Program, Department of Anthropology, Texas A\&M University \\ MKL554@tamu.edu, Chaseoswald@tamu.edu
}

Commission II, WGII/9

KEYWORDS: 3D, Digitization, Ship Models, Photogrammetry Comparisons

\begin{abstract}
:
This study compares the differences between traditional 3D modeling software such as 'Agisoft Metashape' and the recently released smartphone application 'Qlone'. Specifically, this study examines how well each software handles creating 3D renderings of ship models. Comparisons between quality, complexity handling, time, cost, and equipment requirements will be examined to better understand each program's strengths and weaknesses. Ultimately, Agisoft Metashape will demonstrate that it is still the preferred method for most professional and academic use for its high precision accuracy. While Qlone may be a reasonable choice for the average hobbyist or enthusiast due to its availability and relatively low cost.
\end{abstract}

\section{INTRODUCTION}

Advancements in current technology make the creation of $3 \mathrm{D}$ digital models increasingly efficient and decrease the risk associated with the traditional means of acquiring this type of data. But how well does a free phone app compare to more established methods, such as still image photogrammetry and video frame photogrammetry? Can the phone application Qlone provide the same accuracy and quality of the model. A wooden Chinese junk ship model from the Ship Reconstruction Laboratory at Texas A\&M University will be used as the subject for comparing the ability of Qlone and Agisoft Metashape (formerly Photoscan) on ship models. The ship model was scanned with the Qlone application, and compared against two models created in Agisoft Metashape. These models were generated by still and video images which were processed in Agisoft Metashape and the resulting 3D digital models will be compared for accuracy and quality. By analyzing the results of this comparison, the authors hope to determine if Qlone, which is currently free to download and readily available the general public, is a viable option for the digitization of ship models.

Photogrammetry is the science of deriving an accurate 3D point cloud models, allowing both metric and descriptive object information to be gathered from multiple overlapping images. These images can be taken as either still images, or, with proper extraction tools, video footage that then provides individual frames.

The workflow for still image computer vision photogrammetry begins with images taken of the object, in this case a ship model, by a digital camera which is moved all around the stationary object to provide 360 degree coverage. These overlapping images are then inserted into a processing program, in this case Agisoft Metashape, which converts the still images into a digital 3D model. Video frame photogrammetry uses a different source to create the same results. Instead of the camera taking overlapping digital images of the stationary object, the camera is used to take a video of the object, while the camera is moved around the object in a circular motion. Afterward, the frames are digitally extracted from the captured video to create still images. At this point the workflow is essentially the same when photogrammetry is based on captured video, except that still frames are first extracted from video

Qlone is currently a free-to-download app that allows for 3D digitization of physical objects to provides an inexpensive and portable means of photogrammetry. Available on the Qlone website, this app enables both the well-funded professional and the general public, to create 3D digital models.

To utilize the app, the user must first print off a free map, appropriate in scale, which the object to be scanned is placed upon. After opening the application, a dome is projected over the map indicating to the user what angles and coverage must be captured. Using the phone's camera in coordination with the app, the user scans different sections of the object based upon areas indicated by the onscreen dome. These scans are continuously collected through video scan by the application, similar to video recording. Realtime color indication provides the user with a visual reference of what portions have and have not been scanned. The user can then exit the current scan session and either rotate the object or scan the object in an opposite direction to allocate more detail. After the scan is complete the model can be exported for a nominal fee as 3D model in various formats (OBJ, STL, USDZ, GLB, PLY, X3D). 
After completing the 3D render and exporting the model in the desired file format, the user can than import the 3D image into other programs for editing. By conducting this comparison of Qlone against other photogrammetry methods and evaluating the accuracy and quality obtainable with this recently developed application, our plan is to use Qlone in further digitization projects should the results prove satisfactory. These projects could potentially focus on digitally recording existing ship models located within maritime museums. This process would then provide $3 \mathrm{D}$ models that could be studied and analyzed for future reference such as in academic research, public presentations, commercial enterprise and education.

Through comparative methods we hope to demonstrate the differences in quality and cost associated with using different 3D modeling softwares. By highlighting the input and output differentials between traditional photogrammetry softwares like Metashape and newer applications such as Qlone we hope to provide consumers and researchers with a broader knowledge of the 3D modeling applications available to them. Additionally, through this comparative process we aim to determine if $3 D$ ship models produced through the Qlone application are adequate enough for use within the professional and academic sectors.

\section{STATE-OF-THE-ART/RELATED WORK}

3D laser scanners and photogrammetry have been used extensively for recording both ship timbers and the ships themselves, on land and underwater (Tanner 2013, Jones 2013, and Jones 2015). However, the scanning of ship models museums is a subject yet to be studied. Ships in maritime museums have been studied thoroughly. A recent example has been the scanning and 3D modelling of the watercraft collection at the Qatar museum (Cooper et al, 2018). This study hopes to spark an interest in using these neglected materials for both professional and academic study.

\section{METHODOLOGY}

\subsection{Choice of Subjects}

Wooden models of two Chinese junks were chosen as subjects for the study from the Spencer collection of Chinese junk models on display in the Ship Reconstruction Lab at Texas A\&M University. These particular examples were selected based on differences in size, form, and propulsion methods. The Large Junk, see Figure 1, is $42 \mathrm{~cm}$ in length, $9.5 \mathrm{~cm}$ beam and with the mast $47 \mathrm{~cm}$ in height above the surface, this Junk has both a midship cabin and an overhang above the stern. The Small Junk, see figure 2, is $28 \mathrm{~cm}$ in length, 6.5 beam, the stern overhang projects $5.5 \mathrm{~cm}$ in height above the surface. These models provide an array of potential features that will test the abilities of the applications being studied to render accurately.

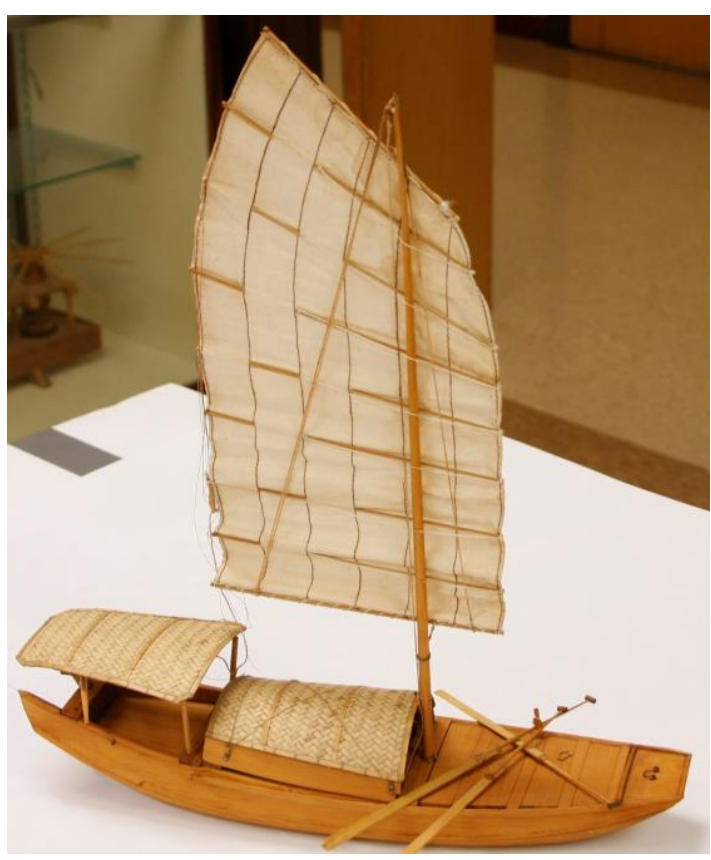

Figure 1. Large Junk Model

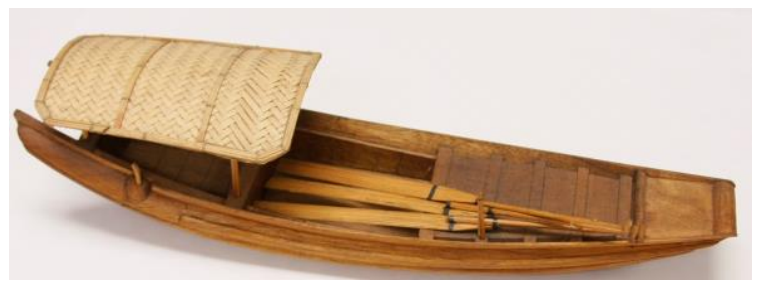

Figure 2. Small Junk Model

\subsection{Image Acquisition}

Images were acquired using either a camera phone or a DSLR camera. Because Qlone is a cell phone application, the authors decided to include images taken using a cell phone camera. This would allow a more accurate comparison, rather then a skewing the results by directly comparing the image quality a cell phone to that of a DLSR camera. The cell phone used to acquire both the Qlone application's 3D model and the image files was a Samsung Galaxy S6.

\subsubsection{Conditions}

All images were taken in the Richard Steffy Ship Reconstruction Laboratory in lighting conditions that would be typically found in a maritime museum. This lighting consisted of overhead fluorescent light fixtures $2.4 \mathrm{~m}$ above floor level. No supplemental or directional lighting were used. These lighting conditions did have adverse effect upon the final model, limiting the resolution on the final photogrammetry models. 


\subsubsection{Qlone}

The Qlone application requires the use of a mat in addition to the use of a cell phone in order to create the digital model. Since the mat is scalable, the first step was to measure the junks used in the study. This resulted in the first issue in using the application, as shown in figure 3 and Figure 4. The application projects a dome bubble above the subject to guide the user's scans. This does not work well with objects that are too long or too narrow or have a large height. Ship models, however, tend to be long and tall. This necessitated an enlarged mat to allow for the mast of the large junk, to be accommodated within the model. This mat limitation would conceivably cause issues with larger models as the radius of the dome is half the width of the mat.

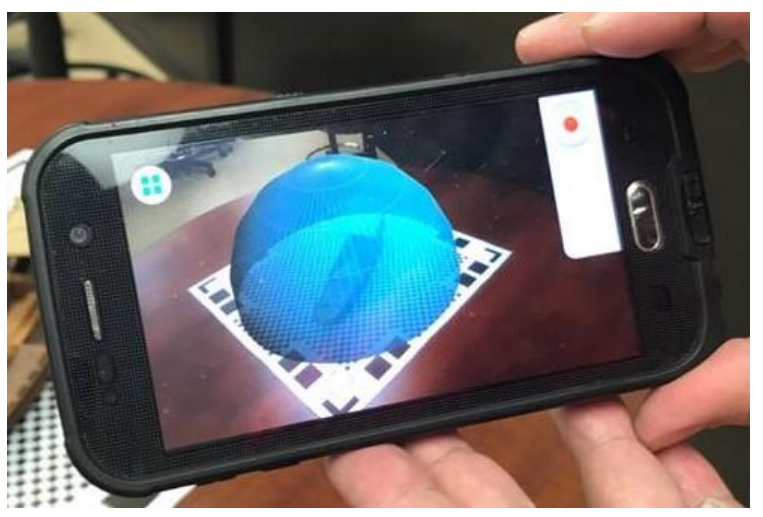

Figure 3. Small Junk Model being scanned with Qlone App on Samsung Galaxy S6 Cell Phone

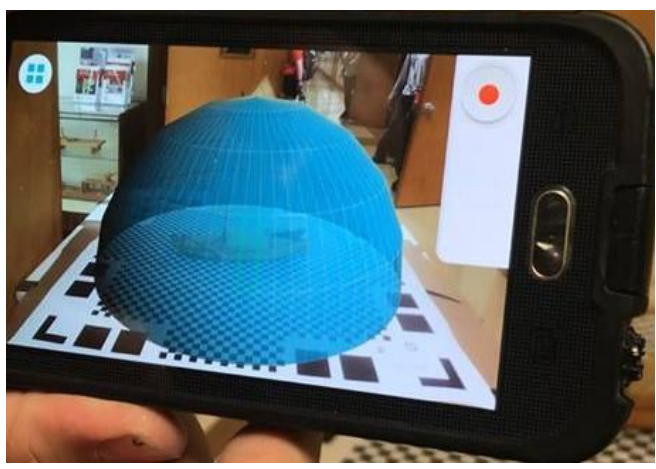

Figure 4. Large Junk Model being scanned with Qlone Application on Samsung Galaxy S6 Cell Phone

\subsection{Agisoft Metashape (Photoscan)}

In order to have a proper comparison between the two softwares it was decided to take photos for photogrammetry using a cell phone camera (Samsung Galaxy S6) in addition to a DLSR camera. The images were taken in a pattern similar to that used with the Qlone model.

\subsection{Image Processing}

Image processing was limited to the use of masking using Adobe Photoshop on both the cell phone and DLSR image files in order for the comparison to be equal. This limitation of course had an effect upon the final models produced in Agisoft Metashape.

\section{COMPARISON OF RESULTS}

The differences between the 3D rendering of the models produced by Qlone and Agisoft Metashape rely heavily on the quality of the images and the complexity of the physical model. For instance, Qlone relies on a continuous video to be taken from a camera phone in which frames from the video are then stitched together to create the model, see Figure 5.

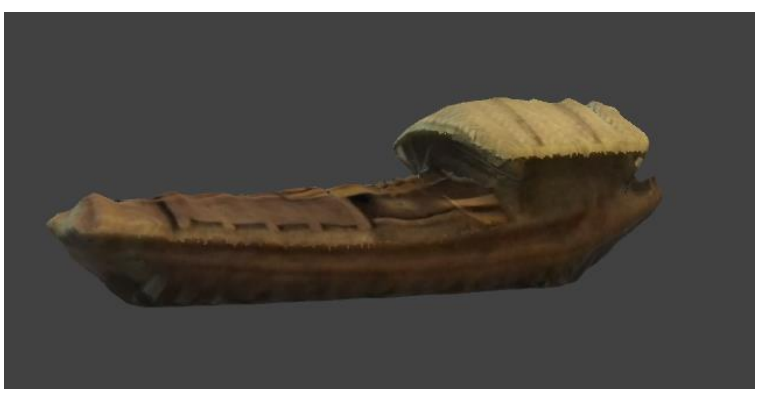

Figure 5. Qlone Model of Small Junk

This method relies heavily on the capabilities of the camera phone, which provides the user with less control of the image settings and lacks the accuracy of a fully focused shot. Thus, the 3D model that is produced using the Qlone method has a less accurate shape and the textures are less detailed than images produced using Agisoft Metahape with images captured from a DSLR camera. Furthemore, it should be noted that the Agisoft Metashape 3D model produced using the same camera phone had superior texture mapping and shape accuracy on the deck of the model than that of the 3D model produced by the Qlone application, see figure $6 \mathrm{a}$ and $6 \mathrm{~b}$. These differences are possibly due to the quality contrast between the use of still images and that of video frames, as well as the differences within the individual softwares.

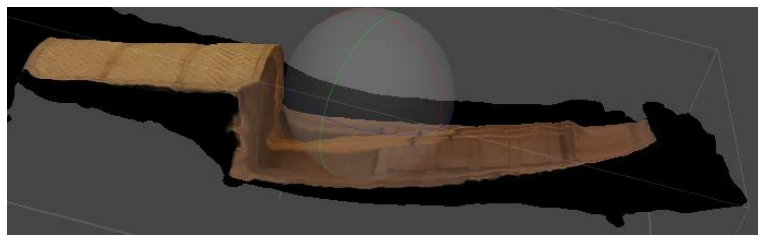

Figure 6a. Small Junk Model creating using Cell phone Images Agisoft MetaShape 


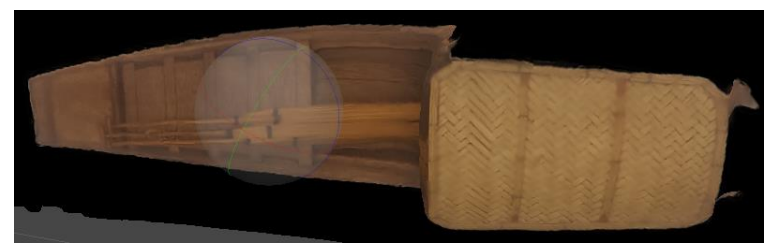

Figure 6b. Small Junk Model creating using Cell phone Images Agisoft MetaShape

When comparing both of the 3D models produced in Agisoft Metashape it is clear that the model using images from a DSLR camera is much more clear and detailed than that produced with a camera phone, most likely due to the better quality of the camera and the higher degree of user input, see figure $7 \mathrm{a}$ and $7 \mathrm{~b}$. Additionally, it should also be that the images taken from the DSLR give the user the ability to choose the file format. As such, TIFF file format was chosen for the DSLR images while the images that were taken by the camera phone were automatically compressed to JPG file format by the cellular device. This compression to JPG file format would account for loss in the image quality.

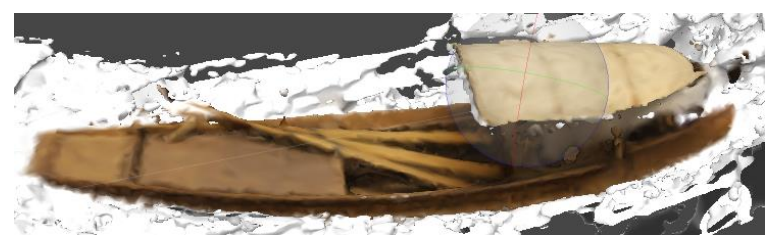

Figure 7a. Small Junk Model creating using DLSR Images and Agisoft MetaShape

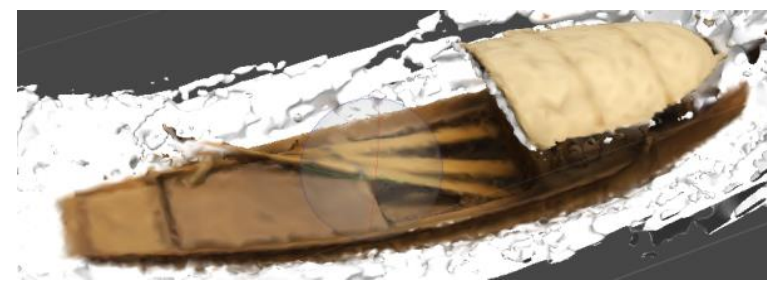

Figure 7b. Small Junk Model creating using DLSR Images and Agisoft Metashape

Another important key trait to pay attention within the creation of the $3 \mathrm{D}$ renderings of these models is the complexity of the ship itself. For instance, the overhang seen on the mast/ sail of the larger junk, see figure 8 and the overhang on the stern of both junks, see figure 9, proved difficult to visualize. All of the methods used in this study had issues visualizing these aspects of the junks. For the larger junk the Qlone application cut off the mast and sail disregarding these components in the final 3D rendering. As for the the overhang on the stern of both junks Qlone filled in the voids between the deck and the overhang with textures borrowed from other portions of the junk. These results demonstrate that the Qlone application ignores overly complex components or small connecting parts,and may actually be incapable of rendering voids in the model. The 3D models using Agisoft Metashape also struggled with these complexities as well but to a lesser extent. While the mast and sail of the larger junk were represented by the 3D models rendered by Agisoft Metashape the associated rigging, however, struggled to be visualized. This problem is most likely due to issues of the lines being visible in images while also being difficult to mask prior to being rendered by Agisoft Metashape. The overhang could not be accurately rendered in Agisoft Metashape due to the inability to capture images of the underside of this feature. The size of the physical model also creates issues for the Qlone application in that larger models require custom enlarged mats in order for the digital dome to cover the entirety of the model. This size issue creates problems for ships that are either large and or disproportionally shaped.

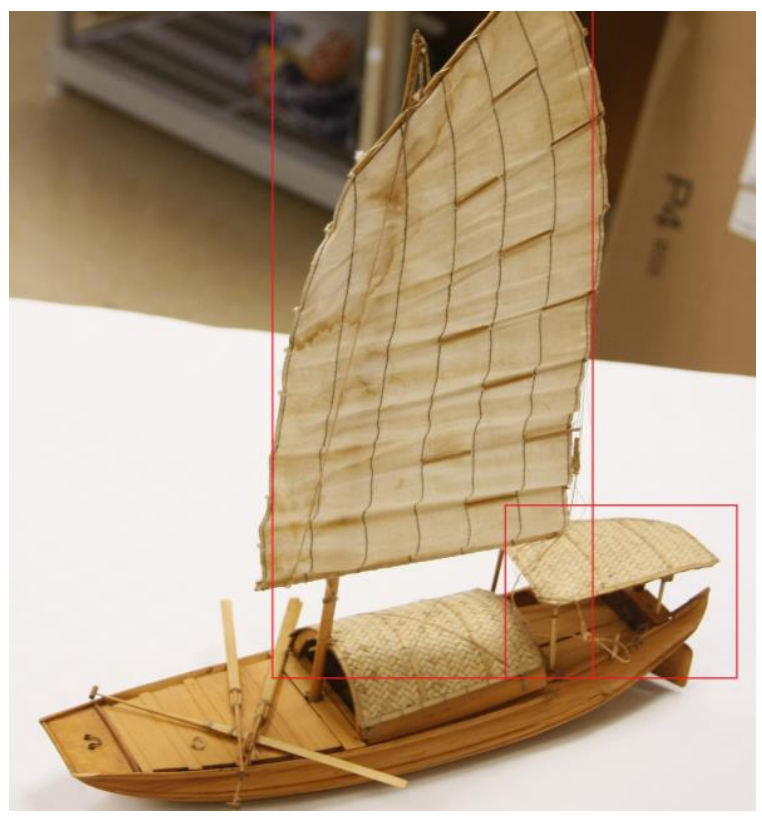

Figure 8. Large Junk with mast /sail and stern overhang outlined in red rectangles.

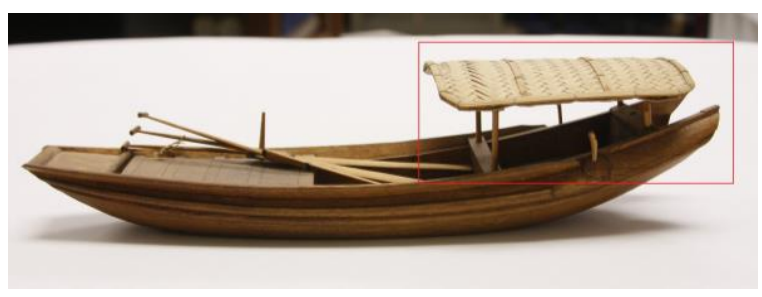

Figure 9. Small Junk with stern overhang outlined in red rectangle.

This study has shown that the quality of the model is incremental to the cost of the capture method being used, see table 1. Qlone Application is free to download onto a persons cell phone and is also free to use. The only cost is the relatively low price payed to download the finished 3D model ( $\$ 1.29$ USD each). In comparison, both the cell phone and DLSR images methods required the use of 
Agisoft Metashape (\$179-3499 USD depending on the edition, although the free trial editions were used for this study) to create the model, in addition to Adobe Photoshop which was required for the image masking. Additionally, an adequate DSLR camera and a computer system that can handle both Adobe Photoshop and Agisoft Metashape can cost anywhere between hundreds and thousands of dollars apiece.

\begin{tabular}{|l|c|c|c|}
\hline Methods & $\begin{array}{l}\text { Quality of } \\
\text { Model }\end{array}$ & Time & Cost \\
\hline Qlone Application & Low & Low & Low \\
\hline $\begin{array}{l}\text { Cell Phone Images, Adobe } \\
\text { Photoshop Masking and } \\
\text { Agisoft Metashape. }\end{array}$ & Med & High & High \\
\hline $\begin{array}{l}\text { DLSR Images, Adobe } \\
\text { Photoshop Masking and } \\
\text { Agisoft Metashape. }\end{array}$ & High & High & High \\
\hline
\end{tabular}

Table 1. Comparison of methods, based upon quality of model to time to cost.

Time is also an important factor to be discussed, both in the sense of how long the entire process takes and how long each 3D model takes to be rendered. The Qlone application process has the advantage in taking less time for both the overall process and rendering time. The entire Qlone application process could easily be completed in under an hour including printing off the mat, capturing the model, and rending the model. Each of these steps takes only minutes in Qlone. Agisoft Metashape process can be a little arduous in comparison. The setup time and photo shoot time roughly took about half an hour to an hour depending on the size of the model. The masking of photos required an additional several hours of work The Agisoft Metashape workflow process then took approximately an hour to complete for each model and is highly dependent on the processing power of the computer being used.

\section{CONCLUSION}

This study has highlighted the comparisons between the use of Qlone and the use of Agisoft Metashape in the creation of $3 \mathrm{D}$ renderings of ship models while also determining the quality of renderings produced by the Qlone application. The comparative analysis herein has lead to the conclusion that while Qlone benefits from low cost and minimal time required, Agisoft Metashape is superior in terms of quality and accuracy. As such, the use of Qlone for creating 3D renderings of ship models is not recommended in terms of professional or academic work, but perhaps may be better suited for the individual enthusiast or hobbyist. While Agisoft Metashape is much more ideal for professional and academic $3 \mathrm{D}$ renderings it may not be as readily available to the individual consumer due to cost and required equipment.

\section{FUTURE WORK}

This study has the potential to be replicated in future work comparing the same 3D modeling applications on other ship models with simple construction and low amounts of detail to see if Qlone could be comparable to Agisoft Metashape under those conditions.

\section{ACKNOWLEDGEMENTS}

The Authors would like to express their thanks for support and advice from the following people;

\author{
Dr. Chris Dostal \\ Dr. Kotaro Yamafune \\ Dr. Luis Vieira De Castro \\ Paul G. Cochran \\ Benjamin Ioset
}

\section{REFERENCES}

Jones, T., 2013. "The Newport Medieval Ship - Timber Recording Manual.”, Newport, Wales, Great-Britain. http://archaeologydataservice.ac.uk/archiveDS/archiveDow nload?t=arch-1563-

1/dissemination/pdf/Newport_Medieval_Ship_Project Tim ber Recording Manual.pdf

Jones, T., 2015 Three-Dimensional Digital Recording and Modelling Methodologies for Documentation and Reconstruction of the Newport Medieval Ship. PhD Thesis submitted at University of Wales Trinity Saint David, Great-Britain.

Metashape, 2019. Agisoft. www.agisoft.com (9 March 2019)

Photoshop Creative Cloud, 2019. Adobe Creative Cloud. www.adobe.com/products/photoshop.html. (9 March 2019)

Qlone, 2017. EyeCue Vision Technologies Ltd. www. Qlone.pro (9 March 2019)

Tanner, P., 2013. 3D Laser Scanning for the Digital Reconstruction and Analysis of a $16^{\text {th }}$-Century Clinker Built Sailing Vessel. Underwater Archaeology Proceedings, pp. 137-149.

Yamafune, K. 2016. Using Computer Vision Photogrammetry (Agisoft Photoscan) to Record and Analyze Underwater Shipwreck Sites 